\title{
Antidote bei Vergiftungen 2007
}

\section{H. Kupferschmidt*}

Für die Arbeitsgruppe «Antidota» des Schweizerischen Toxikologischen Informationszentrums (STIZ) und der Gesellschaft Schweizerischer Amts- und Spitalapotheker (GSASA)
Korrespondenz:

Dr. med. Hugo Kupferschmidt

Schweizerisches Toxikologisches Informationszentrum

Freiestrasse 16

CH-8032 Zürich

Tel. 0442516666

Fax 0442528833

hkupferschmidt@toxi.ch
Die Arbeitsgruppe «Antidota» des Schweizerischen Toxikologischen Informationszentrums (STIZ) und der Gesellschaft der Schweizerischen Amts- und Spitalapotheker (GSASA) hat die Schweizerische Antidotliste überarbeitet und im BAG-Bulletin 2007/5 veröffentlicht. Sie gibt Aufschluss über die Art der Antidote und deren Verfügbarkeit. Es werden nur Substanzen in die Liste aufgenommen, die nicht bereits zur Standardausrüstung der öffentlichen Apotheken und Spitalapotheken gehören und dabei folgende Kriterien erfüllen: 1. Die Substanz wird als klassisches Antidot angewendet; 2. Ein Medikament, das nicht generell im Spital verfügbar ist, findet als Antidot Anwendung; 3. Die Anwendung als Antidot erfordert grössere Mengen als die, die für den therapeutischen Einsatz im Spital normalerweise vorrätig sind, und 4. die Anwendung als Antidot ist wenig bekannt. Es wird keine Vollständigkeit, sondern Sicherheit bezüglich des effektiven Vorhandenseins der ausgewählten Präparate angestrebt. Dieses Konzept der flächendeckenden Antidotaverteilung in der Schweiz wurde 1986 von der Gesundheitsdirektorenkonferenz genehmigt und 2005 erneut bestätigt. Der Stiftungsrat des Schweizerischen Toxikologischen Informationszentrums begrüsst dieses Notfallversorgungskonzept und unterstützt dessen Aufrechterhaltung mit der vorliegenden Antidotliste, die die Grundlage für dieses Versorgungskonzept bildet.

Das Schweizer Antidotarium ist in drei sich ergänzende Sortimente gegliedert, wobei Vergiftungshäufigkeit, Ort der Verwendung und logistische Kriterien für die Zuteilung entscheidend sind. Ein Grundsortiment ist generell in allen Akutspitälern und öffentlichen Apotheken vorhanden, ein Zusatzsortiment lediglich in Regionalzentren, die grossen Spitälern angegliedert sind (Tab. 1). Diese Sortimente bilden die Basis für eine optimale ärztliche Versorgung von toxikologischen Notfallsituationen.

\section{Verfügbarkeit der Antidote}

Mehr als die Hälfte der Präparate auf der Antidotliste ist nicht regulär zugelassen und muss auf der Grundlage des Art. 36 der Arzneimittelbewilligungsverordnung (AMBV) direkt von den Herstellern oder über Grenzapotheken importiert werden. Da viele Antidote wirtschaftlich kaum interessant sind, und weil die Hürden für eine ordentliche Zulassung hoch sind, ist es unrealistisch, zu erwarten, dass sich Hersteller in der Schweiz um eine Zulassung bemühen. Schlimmer noch, unter dem wirtschaftlichen Druck verschwinden immer mehr dieser unverzichtbaren Medikamente vom Markt. Dass die Verantwortung für die Versorgungssicherheit (Kantone) und die Verantwortung für die Arzneimittelsicherheit (Swissmedic) nicht an derselben Stelle vereint wahrgenommen werden, macht eine einfache und zuverlässige Verfügbarkeit der Antidote zusätzlich schwierig. Die Beschaffung dieser Präparate im Ausland ist längerfristig nicht garantiert, und im Inland wird sich ohne behördliche Unterstützung niemand freiwillig an die Herstellung dieser Präparate machen. Hier hilft langfristig nur eine politische Entscheidung.

Aufgrund der gesetzlichen Vorschriften (die revidierte Institutsverordnung zum Heilmittelgesetz ist seit dem 1. Oktober 2006 in Kraft) gibt es grundsätzlich drei Möglichkeiten, ausserhalb des ordentlichen Zulassungsverfahrens Heilmittel in Verkehr zu bringen:

1. Es können Swissmedic Arzneimittelmonographien zur Pharmakopöe eingereicht werden, aufgrund deren Apotheken kleine Mengen herstellen dürfen (Art. 15 VAZV). Für Herstellung und Vertrieb müssen entsprechende Bewilligungen vorliegen.

2. Ebenfalls möglich sind vereinfachte Zulassungen gemäss Art. 14 HMG. Dazu ist ein Antrag an Swissmedic mit einer entsprechenden Dokumentation nötig; die pharmakologische und toxikologische Dokumentation bekannter Wirkstoffe, wie diejengen in der Antidotliste, ist mit Literaturreferenzen machbar.

3. Der Direktimport gemäss Verordnung über die Bewilligungen im Arzneimittelbereich (AMBV Art. 36) ist nach wie vor gestattet, wenn kein vergleichbares Produkt in der Schweiz im Handel ist. Ordentlich in der Schweiz zugelassene Antidote werden auf dem auch für andere Arzneimittel üblichen Weg beschafft.

Das Netzwerk der Schweizerischen AntiveninDepots ANTIVENIN-CH [1] hat 2005/2006 13mal Antivenine ausgeliefert. Im Juni 2006 wurde wegen des Verdachts, es könnten eine Anzahl exotischer Giftschlangen entwichen sein, 
ein Notfallplan für den Kanton Bern erstellt. Das STIZ registrierte 2005/2006 105 Anfragen zu Schlangenbissen, $76 \mathrm{zu}$ einheimischen, $29 \mathrm{zu}$ exotischen Schlangen. In 53 Fällen handelte es sich um Giftschlangen, 16 davon in Haltung (Kupferkopf, Gabunviper, Malayische Grubenotter, Klapperschlangen, Kobras, Taipan, Mambas, Ceylon-Lanzenotter, Sandrasselotter, MacMahon-Viper, Levanteotter). Fünf Bisse von Giftschlangen verliefen ohne Symptome, 15 mit leichten, 9 mit mittelschweren und 4 mit schweren Symptomen. Todesfälle gab es keine (von den übrigen liegen keine Verlaufsbeobachtungen vor).

\section{News 2007}

1. Der Bestand an Antidoten in den Regionalzentren wird im Lauf des Jahres 2007 auf der Website der GSASA einsehbar sein (www. gsasa.ch).

2. Das Kalziumglukonatpräparat der Firma Streuli (Glucocalcium Streuli) ist nicht mehr im Han-

\begin{tabular}{|c|c|c|c|}
\hline & Ort & Spital & Telephonnummer \\
\hline 1 & Aarau & Kantonsspital Aarau AG & 0628384141 \\
\hline 2 & Basel & Universitätsspital & 0612652525 \\
\hline 3 & Bellinzona & Ospedale San Giovanni & 0918119111 \\
\hline 4 & Bern & Inselspital & 0316322111 \\
\hline 5 & Biel & Regionalspital & 0323244860 \\
\hline 6 & Chur & Rätisches Kantons- und Regionalspital & 0812566111 \\
\hline 7 & Delémont & Hôpital régional & 0324212121 \\
\hline 8 & Fribourg & Hôpital cantonal & 0264267111 \\
\hline 9 & Genève & Hôpital universitaire (HUG) & 0223723311 \\
\hline 10 & Interlaken & Regionalspital & 0338262626 \\
\hline 11 & Lausanne & CHUV & 0213141111 \\
\hline 12 & Lugano & Ospedale civico & 0918116111 \\
\hline 13 & Luzern & Kantonsspital & 0412051111 \\
\hline 14 & Münsterlingen & Kantonsspital & 0716861111 \\
\hline 15 & Neuchâtel & Hôpital Pourtalès & 0327133000 \\
\hline 16 & Samedan & Spital Oberengadin & 0818518111 \\
\hline 17 & St. Gallen & Kantonsspital & 0714941111 \\
\hline 18 & Schaffhausen & Kantonsspital & 0526343434 \\
\hline 19 & Schwyz & Spital & 0418184111 \\
\hline 20 & Sion & Zentralinstitut der Walliser Spitäler & 0276034000 \\
\hline 21 & Solothurn & Bürgerspital & 0326273121 \\
\hline 22 & Winterthur & Kantonsspital & 0522662121 \\
\hline 23 & Zürich & Universitätsspital & 0442552111 \\
\hline 24 & Zürich & Stadtspital Triemli & 0444662220 \\
\hline 25 & Zug & Kantonsspital & 0417097660 \\
\hline
\end{tabular}

del. Weiterhin erhältlich ist jedoch KalziumSandoz. Das Kalziumglubionat kann wie Kalziumglukonat verwendet werden.

3. Diphtherie-Antitoxin ist neu in der Armeeapotheke verfügbar und kann über das STIZ bezogen werden (gleich wie Botulinus-Antitoxin).

4. Hochdosiertes Insulin in Kombination mit Glukose wird heute regelmässig bei Vergiftungen mit Kalziumkanalblockern eingesetzt [2-6]. Erste Hinweise über die Wirksamkeit der sogenannten HIE (Hyperinsulinaemia/ Euglycaemia) stammen aus Tiermodellen [7, 8], wobei der genaue Wirkungsmechanismus bisher unklar ist. Eine Rolle zu spielen scheint einerseits der positiv-inotrope Effekt des Insulins. Andererseits soll durch die Insulinwirkung die Kohlehydratverwertung des Myokards verbessert werden. Da es sich um eine experimentelle Therapie handelt, wird geraten, erst bei fehlendem Erfolg der bewährten Therapiemassnahmen Insulin als Bolus von 1,0 IU/kg KG intravenös, gefolgt von $0,5 \mathrm{IU} / \mathrm{kg} / \mathrm{h}$ intravenös zu verabreichen. Zur Gewährleistung der Euglykämie muss gleichzeitig Glukose 20-30 g/h (0,5-1,0 g/kg KG/h) intravenös gegeben werden, initial ebenfalls als Bolus von $0,5-1 \mathrm{~g} / \mathrm{kg}$. Eine engmaschige Kontrolle von Plasmaglukose und Serumkalium ist unumgänglich.

5. Zur Behandlung des Serotoninsyndroms wird (neben der supportiven Therapie) oft der Serotoninantagonist Cyproheptadin als Antidot genannt [9-12]. Daneben werden auch andere $5-\mathrm{HT}_{2 \mathrm{~A}}$-Antagonisten wie Olanzapin (10 mg sublingual) oder Chlorpromazin (50$100 \mathrm{mg}$ intramuskulär) eingesetzt [13-15]. Es ist bis heute nicht klar, welches die Therapieoption der ersten Wahl ist.

6. Digitalis-Antidot ${ }^{\circledR}(80 \mathrm{mg})$ wird im Lauf des Jahres 2007 durch DigiFab ${ }^{\text {TM }}$ (40 mg) ersetzt. Laut einer Mitteilung des Herstellers von DigiFab $^{\mathrm{TM}}$, Protherics PLC, hat die Firma die Marktrechte für Digitalis-Antidot ${ }^{\circledR}$ von Roche erworben; Proreo Pharma AG wird das Präparat in der Schweiz registrieren und vertreiben.

7. $\mathrm{CaNa}_{2}$-EDTA ist nur noch in Form des Präparates der Laboratoires SERB verfügbar.

Die Antidotliste erscheint jedes Jahr regelmässig im BAG-Bulletin und ist auch auf dem Internet einsehbar über www.toxi.ch oder www.pharmavista.net. Dort sind zusätzlich Links zu weiterführender Information (Antidotmonographien, Fachinformationen) vorhanden. 


\section{Literatur}

1 Kupferschmidt H. Antidote bei Vergiftungen 2004. Gründung des Netzwerks Schweizerischer Schlangenserum-Depots. Schweiz Ärztezeitung. 2004; 85(26):1378-9.

2 Yuan TH, Kerns W, et al. Insulin-glucose as adjunctive therapy for severe calcium channel antagonist poisoning. J Toxicol Clin Toxicol. 1999; 37:463-74.

3 Boyer EW, Shannon M. Treatment of calciumchannel-blocker intoxication with insulin infusion. NEJM. 2001;344(22):1721-2.

4 Rasmussen L, Husted SE, et al. Severe intoxication after an intentional overdose of amlodipine. Acta Anaesthesiol Scand. 2003;47:1038-40.

5 Salhanick SD, Shannon MW. Management of calcium channel antagonist overdose. Drug Saf. 2003;26:65-79.

6 Coleman GM, Gradinac S, et al. Efficacy of metabolic support with glucose-insulin-potassium for left ventricular pump failure after aortocoronary bypass surgery. Circulation. 1989;80:191-6.

7 Kline JA, Tomaszewski CA, et al. Cardiovascular toxicity induced by verapamil in the anesthetized canine. J Pharmacol Exp Ther. 1993;267:744-50.
8 Reikeras Kline JA, Gunnes P, et al. Haemodynamic effects of high doses of insulin during acute left ventricular failure in dogs. Eur Heart J. 1985; 6:451-7.

9 Graudins A, Stearman A, Chan B. Serotonin syndrome successfully treated with cyproheptadine. J Toxicol Clin Toxicol. 1997;35:539-40.

10 Graudins A, Stearman A, Chan B. Treatment of the serotonin syndrome with cyproheptadine. J Emerg Med. 1998;16:615-9.

11 Lappin RI, Auchincloss EL. Treatment of serotonin syndrome with cyproheptadine. NEJM. 1994;331:1021-2.

12 Mills KC. Serotonin syndrome: a clinical update. Crit Care Clin. 1997;13:763-83.

13 Gillman PK. The serotonin syndrome and its treatment. J Psychopharmacol. 1999;13:100-9.

14 Boyer EW, Shannon M. The serotonin syndrome. NEJM. 2005;352:1112-20.

15 Claassen JAHR. The serotonin syndrome [letter]. NEJM. 2005;352:2455 